\title{
A Facile One-Pot Operations of Reduction and Allylation of Nitrobenzaldehydes Mediated by Indium and Their Applications ${ }^{\dagger}$
}

\author{
Yong Seo Cho, Kyung Ho Kang, Joo Hwan Cha, Kyung Il Choi, Ae Nim Pae, Hun Yeong Koh, and Moon Ho Chang \\ Biochemicals Research Center. Norea Institute of Science and Technologv, P.O. Box I3I Cheongrvang, Seoul 130-650, Korea \\ Receivedilpril 2. 2002
}

\begin{abstract}
Various nitrobenzaldehydes were simultaneously ally lated and reduced using indium in the presence of $\mathrm{HCl}$ in aqueous media to give compounds having both functionality of homoallylic alcohol and aromatic anine. Sequential protection of the amino group and oxidation of the anilinyl homoally lic alcohol provided useful precursors of heterocyclic compounds such as dihydroindolones and dihydroquinolones, which could be efficiently sy nthesized through intramolecular cyclization reaction.
\end{abstract}

Key Words : Simultancous reduction-ally lation. Indium. Aqucous media

\section{Introduction}

Heterocycles such as quinolone. dily droquinolone. indole. and dihydroindolone have been found in a variety of the biologically active compounds. Development of efficient synthetic protocol for these compounds is very important in organic and medicinal chemistry: Both metal-mediated allylation reactions ${ }^{1}$ and reduction reactions of nitro group ${ }^{2.3}$ are important processes frequently met in organic synthesis. Recently; we found that indium can mediate the reduction of nitro group to amine in the presence of $\mathrm{HCl}$ in aqueous $\mathrm{THF}^{4}$ Combining these two actions of indium, we have performed one-pot reduction and allylation reaction of nitro and aldehyde groups. Herein we report simultaneous reduction-allylation reactions of nitro and aldelyde groups of various nitrobenzaldelydes 1 in aqueous media to give anilinyl homoallylic alcohols $\mathbf{2}$ under a mild reaction condition (Scheme 1). The anilinyl homoally lic alcohols 2 could successfully transform into dilyydroindolones 6 and dilyydroquinolones $7 \mathrm{by}$ using base without protection for the intramolecular cyclization.

\section{Results and Discussion}

The results of the reactions of various o-nitrobenzalde-<smiles>[R]c1ccc(C=O)c([N+](=O)[O-])c1</smiles><smiles>[R7]C=C([R2])CBr</smiles>

1

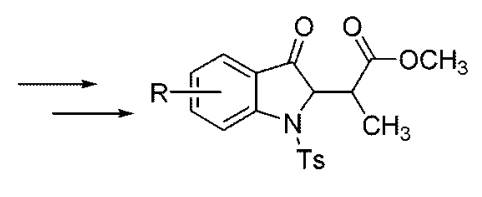

6<smiles>[R][X]1ccc(N)c(C(O)C([R7])C([R2])=C)c1</smiles>

2

or<smiles>[R][X]c1ccc2c(c1)N([3H])C([R2])(C)C([R])C2=O</smiles>

7
Scheme 1

${ }^{\mp}$ This paper is dedicated to the late Professor Sang Chul Shim. hydes were summarized in Table 1 . The first three nitrobenzaldehydes were converted to the corresponding anilinyl homoally lic alcohol 2 in moderate yields (Entry 1-12). The 6-nitropiperonal in entry 13-16 gave low yields suggesting an unfavorable effect of electron-releasing substituents and labile moiety in acidic condition. In case of 3-methoxy-2nitrobenzaldehyde (Entry 17-20). only the allylation products 3a-3d were obtained in $88-94 \%$ yields. probably due

Table 1. Ally lation-Reduction Reactions of $o$-Nitrobenzaldehyde

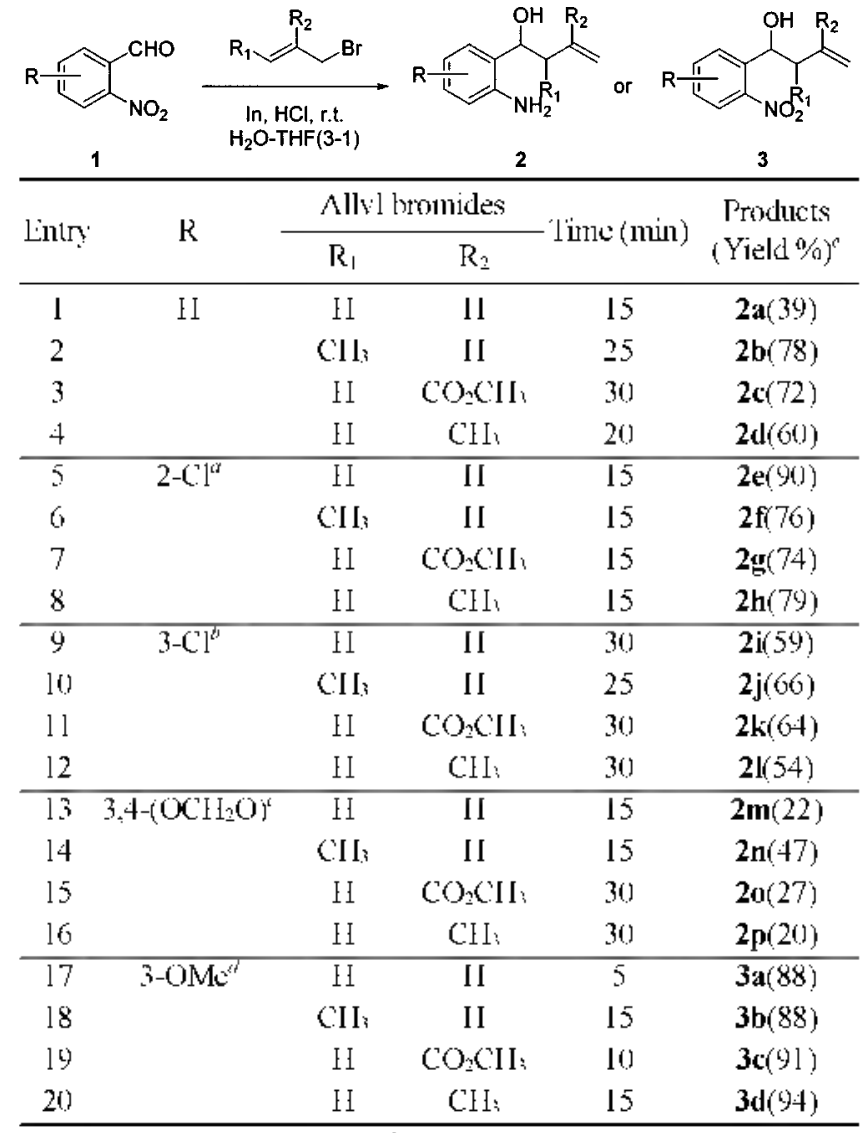

a-Chloro-6-nitrobenzaldelyde: "3-Chloro-2-nitrobenzaldelivde: "6-Nitropiperonal: "3-Methoxy-2-nitrobenzaldelyde: "Isolated vield. 
Table 2. Intramolecular Cyclization of $5 a-5 c$ in the Presence of Bases

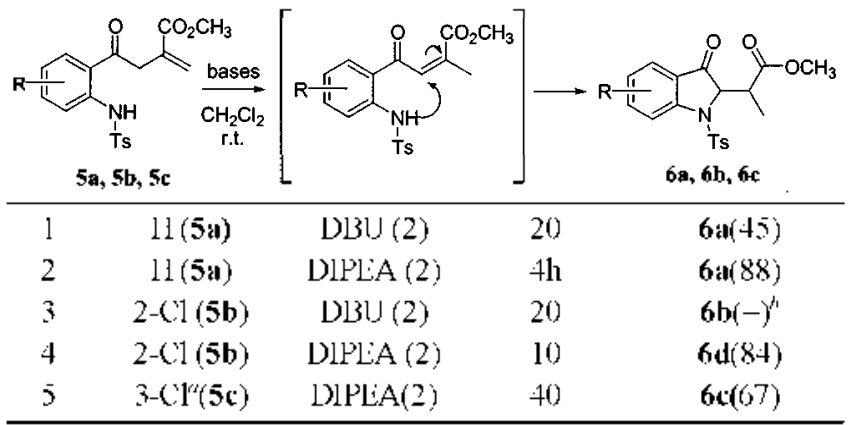

"Isolated yicld: "No product was obtained

to the clectron donating effect of the methoxy group at the 3position.

Simultancous reactions of ally lation and reduction could be accomplished in the presence of $\mathrm{HCl}$ by indium. Withoul $\mathrm{HCl}$. only the allylation of aldelyde group only proceded indicating that $\mathrm{HCl}$ made a crucial role for the reduction. For example. the reaction between 3-chloro-2-nitroben/aldehyde and allyl bromide by indium without $\mathrm{HCl}$ gave the only ally lated product at room temperature for $12 \mathrm{~h}$. along with $40 \%$ of the recovered starting material.

Various anilinyl homoallylic alcohols 2 gencrated were protccted by tosylation with $\mathrm{TsCl}$ at $0^{\circ} \mathrm{C}$ in pyridine for 4 h$12 \mathrm{~b}$ to alford the sulfonamides 4 in $62 \%$ to $97 \%$ viclds. Sulfonamides 4 were oxidized with using PCC at rt for 4 h12 h to give $5 \mathbf{a}-\mathbf{5 i}$ in $44 \%$ to $91 \%$ yiclds (Scheme 2 ).

We carried out the intramolecular cyclization of $\mathbf{5}$ a. which has elcctron-defficicnt methoxycarbonyl moicty with $2 \mathrm{cq}$. of DBU as shown in entry 1 of Table 2. The 1.4-addition to $\alpha . \beta$-unsaturated ester after migration of double bond by DBU occurred to give the five-membered dihydroindolone 6a in moderate yield $(45 \%)$. In case of $5 \mathbf{b}$. no product was<smiles>[R][Y]1ccc(N)c(C(O)C([R2])C([R])=C)c1</smiles>

$$
\begin{aligned}
& \underset{\text { TsCl }}{\text { pyridine }} \\
& 60 \sim 90 \%
\end{aligned}
$$

2

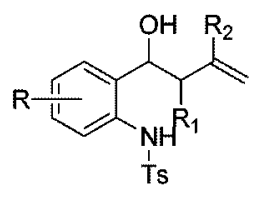

4

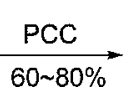<smiles>[Y5]Nc1cc[R]([R])cc1C(=O)C([R7])C([R2])=C</smiles>

5
$4 a(78 \%): \mathrm{R}=\mathrm{H}, \mathrm{R}_{1}=\mathrm{H}, \mathrm{R}_{2}=\mathrm{CO}_{2} \mathrm{CH}_{3}$ : $4 \mathrm{~b}(76 \%): \mathrm{R}=2-\mathrm{Cl}, \mathrm{R}_{1}=\mathrm{H} . \mathrm{R}_{2}=\mathrm{CO}_{2} \mathrm{CH}_{3}$ : $4 \mathbf{c}(62 \%): \mathrm{R}=3-\mathrm{Cl}, \mathrm{R}_{1}=\mathrm{H}_{1} \mathrm{R}_{2}=\mathrm{CO}_{2} \mathrm{CH}_{3}$; $\mathbf{4 d}(80 \%): \mathbf{R}=\mathbf{H}_{1}, \mathbf{R}_{1}=\mathbf{R}_{2}=H_{\text {; }}$ $4 e(97 \%): R=3-C_{1}, R_{1}=R_{2}=H$ : $4 f(87 \%): R=H, R_{1}=C_{3}, R_{2}=H$; $4 \mathrm{~g}(92 \%): \mathrm{R}=3-\mathrm{Cl}, \mathrm{R}_{1}=\mathrm{CH}_{3}, \mathrm{R}_{2}=\mathrm{H}$; $4 h(89 \%): R=H_{1} R_{1}=H . R_{2}=C_{3}$ i $4 i(94 \%): R=3-C l, R_{1}=H, R_{2}=\mathrm{CH}_{3}$.
$5 a(84 \%): R=H, R_{1}=H, R_{2}=C_{2} C_{3}$ : $5 \mathrm{~b}(65 \%): \mathrm{R}=2-\mathrm{Cl}, \mathrm{R}_{\mathbf{1}}=\mathrm{H}, \mathrm{R}_{2}=\mathrm{CO}_{2} \mathrm{CH}_{3}$; $5 c(85 \%): \mathrm{R}=3-\mathrm{Cl}, \mathrm{R}_{1}=\mathrm{H}, \mathrm{R}_{2}=\mathrm{CO}_{2} \mathrm{CH}_{3}$; $5 d(73 \%): R=H, R_{1}=R_{2}=H_{;}$ $S e(86 \%): R=3-C l, R_{1}=R_{2}=H$; Sf $(44 \%): \mathrm{R}=\mathrm{H}, \mathrm{R}_{1}=\mathrm{CH}_{3}, \mathrm{R}_{2}=\mathrm{H}$; $5 \mathrm{~g}(70 \%): \mathrm{R}=3-\mathrm{Cl}, \mathrm{R}_{1}=\mathrm{CH}_{3}, \mathrm{R}_{2}=\mathrm{H}$; $5 h(71 \%): R=H, R_{1}=H . R_{2}=C_{3}$; 5i( $(77 \%): \mathrm{R}=3-\mathrm{Cl}, \mathrm{R}_{1}=\mathrm{H}, \mathrm{R}_{2}=\mathrm{CH}_{3}$
Table 3. Intramolecular Cvclization of 5d-5i in the Presence of DBU (2 eq.)

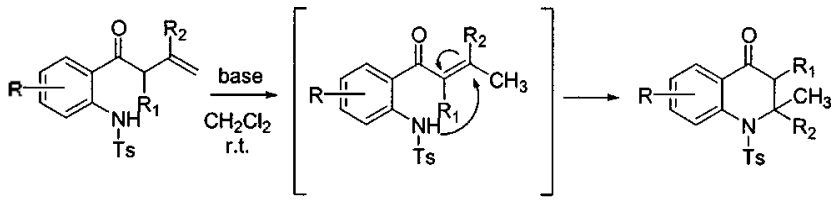

\begin{tabular}{|c|c|c|c|c|c|}
\hline \multicolumn{2}{|c|}{$5 d-5 i$} & \multirow[b]{2}{*}{$\mathrm{R}_{\mathrm{l}}$} & \multirow[b]{2}{*}{$\mathrm{R}_{2}$} & \multirow[b]{2}{*}{ Time (mint) } & $7 d-71$ \\
\hline F.ntry & $\mathrm{R}$ & & & & $\begin{array}{l}\text { Products } \\
\text { (Yicld \%) }\end{array}$ \\
\hline 1 & 11 & II & 11 & 30 & $7 \mathbf{d}(77)$ \\
\hline 2 & $3-C !$ & I & 11 & 30 & $7 e(78)$ \\
\hline 3 & 11 & $\mathrm{Cll}_{3}$ & 11 & 90 & $7 f(91)$ \\
\hline 4 & $3-C !$ & $\mathrm{Cll}_{3}$ & 11 & 10 & $7 \mathrm{~g}(86)$ \\
\hline 5 & 11 & II & $\mathrm{CH}_{3}$ & $50 h^{l 1}$ & $7 \mathbf{h}(70)$ \\
\hline 6 & 3-C'1 & II & $\mathrm{CH}_{3}$ & $48 h^{l}$ & $7 \mathrm{i}(76)$ \\
\hline
\end{tabular}

"Tsolated sicld: "Reaction mixture was retluxed at 40 'C' in sealed tube.

obtained (entry 3) that might be duc to the strong basicity of DBU.

The intramolecular cyclizations were improved by using DIPEA ( $\left.i-\mathrm{Pr}_{2} \mathrm{NEt}\right)$. Threc substrates $\mathbf{5} \mathbf{a}-\mathbf{5} \mathbf{c}$ smoothly procecded to give the corresponding dihy droindolone rings $6 \mathbf{6}-\mathbf{6 c}$ in $67-88 \%$ yiclds by using DIPEA (Tablc 2) through the migration of the double bond under the mild basic condition.

The intramolccular cyclizations of sulfonamides $\mathbf{5}(\mathbf{i}-\mathbf{5} \mathbf{i}$ generated from the other ally] bromides such as allyl bromide. 3-bromo-2-methylpropenc. and crotyl bromide were also studicd. As shown in Table 3. the dihydroquinolone rings could be oblaincd by Michacl addition reaction of $\alpha . \beta-$ unsalurated ketones in situ generated by using DBU. Intramolccular cyclizations of $\mathbf{5}(\mathbf{l}-\mathbf{5} \mathrm{g}$ smoothly procecded to give $7 \mathrm{~d}-7 \mathrm{~g}$ at room temperature.

In case of $\mathbf{5 h}$ and $\mathbf{5 i}$. which compounds have methylpropenyl moicty (cntry 5.6 Table 3). treatment with DBU at room temperature for 24 h gave both the cyclized product $7 \mathrm{~h}$ and $7 \mathbf{i}$ and the migrated intermediate $8 \mathbf{h}$ and $8 \mathbf{i}$ in a ratio of $1: 1.2$ as shown in Scheme 3. These cyclizations could be completed to the corresponding product $7 \mathrm{~h}$ and $7 \mathrm{i}$ at $40^{\circ} \mathrm{C}$ in sealed tube for $48 \mathrm{~h}-50 \mathrm{~h}$. respectively.

In conclusion, various substituted nitrobeızaldellydes underwent a simultaneous ally lation and reduction reaction mediated by indum in the presence of $\mathrm{HCl}$ in aqueous media. Sequential protection and oxidation reactions of various anilinyl honoally lic alcohols provided useful precursors for the 5- and 6membered heterocyclic compounds such as dilydroindolone or dilydroquinolone rings which could be efficiently obtailled by intramolecular cyclization using DIEA or DBU.

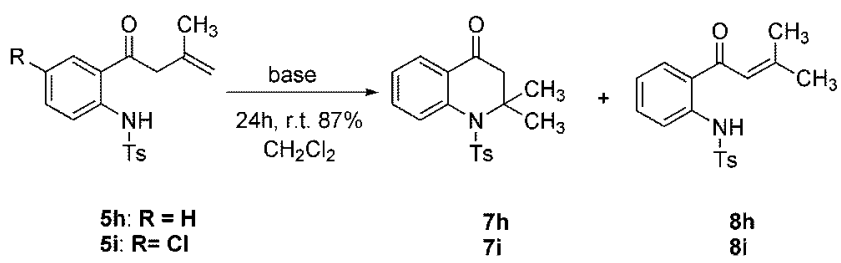




\section{Experimental Section}

All the commercially available reagents were obtained from Aldrich. Fluka, and generally used without further purification. Anhydrous procedures were performed with purificd solvents. Reaction was performed under nitrogen atmosphere.

${ }^{\mathrm{l}} \mathrm{H}$ NMR and ${ }^{13} \mathrm{C}$ NMR spectra were oblained on a Varian Gemini 300 and Bruker Advance 300 spectrometers. Nuclear magnetic resonance spectra were acquired at 300 (or 200 ) $\mathrm{MH} /$ for ${ }^{1} \mathrm{H}$. and $75 \mathrm{MH} /$ for ${ }^{1} \mathrm{C}$. Infrared spectra were oblained on a Perkin Elmer l6FPC FT-lR spectrometer using $\mathrm{KBr}$ pellet. $\mathrm{CHCl}_{3}$ or neat. GC/MSD were oblained on a Hewlet Packard 5890. HRMS spectra were obtained on a JMS-700 mass spectrometer (Jcol). Analytical thin layer chromatographics (TLC) were carricd out on precoatcd silica gel plates (Merck Kicselgel 60)254. layer thickness $0.25 \mathrm{~mm}$ ). Flash column chromatographies were conducted with silica gel grade 230-400 mesh (Merck Kiesegel 60) Art 9385)

Representative procedure for a simultaneous allỵlation and reduction reactions.

Synthesis of 1-(2-aminophenỵl)but-3-en-1-ol (2a): 2Nitroben $\%$ aldehy de (40.5 mg. $0.27 \mathrm{mmol}$ ). indium (184 mg. $1.60 \mathrm{mmol})$ and allyl bromide $(34.6 \mu \mathrm{L}, 0.4 \mathrm{mmol})$ were dissolved in aqueous solution ( $\mathrm{H}_{2} \mathrm{O}-\mathrm{THF} . \mathrm{v} / 3: 1.3 \mathrm{~mL}$ ) and concentraled $\mathrm{HCl}(37 \%, 180 \mathrm{~mL})$ was added dropwisc to the reaction mixture. After stirring for $5 \mathrm{~min}$ at room temperature. the reaction mixture was extracted with cthylacctatc $(10 \mathrm{~mL} \times 2)$ and sequentially washed with saturated $\mathrm{NaHCO}_{3}$. water. and brine. The combined organic layers were dricd $\left(\mathrm{MgSO}_{4}\right)$. concentrated in wiculo. and purilicd by column chromatography to give product (17.6 mg. $39 \%),{ }^{1} \mathrm{H}$ NMR (30) MH $\left.\% \mathrm{CDCl}_{3}\right) \delta 2.56-2.75(2 \mathrm{H} . \mathrm{m}) .3 .90(2 \mathrm{H}$. brs). $4.68(1 \mathrm{H} . \mathrm{dd} . J=5.43 \mathrm{H} / . J=8.49 \mathrm{H} /$ ). 5.14 ( $\mathrm{lH} . \mathrm{d} . J=$ $5.58 \mathrm{H} \%$ ). $5.19(1 \mathrm{H} . \mathrm{d} .13 .9 \mathrm{H} \%) .5 .86(\mathrm{lH} . \mathrm{m}) .6 .65(\mathrm{lH} . \mathrm{d} . J$ $=7.83 \mathrm{H} \%) .6 .73(\mathrm{HH}, \mathrm{l} . J=6,6 \mathrm{H} \%) .7 .04(2 \mathrm{H}$. ovcrlap m): ${ }^{1.3} \mathrm{C}$ NMR (75 MHz. CDCl 3$) \delta 41.3,74.3,118.1,119.5 .127 .7$, 128.8. 129.9. 135.2: IR (neat. cm ${ }^{-1}$ ) 3714. 3415. 3046. 2917; MS(EI) Anal. Calcd for $\mathrm{C}_{10} \mathrm{H}_{13} \mathrm{NO}: 163.09$. Found: 163.00 .

1-(2-Aminophenyl)-2-methylbut-3-en-1-ol (2b): 'H NMR $\left(300 \mathrm{MHz}, \mathrm{CDCl}_{3}\right) \delta 1.16(3 \mathrm{H}, \mathrm{d}, J=6.66 \mathrm{~Hz}) .2 .28(1 \mathrm{H}$. II). 3.7 (2H. brs). 4.45 (1H. d. $J=7.47 \mathrm{~Hz}) .4 .96(1 \mathrm{H}, \mathrm{d} . J=$ $12.0 \mathrm{~Hz}) .5 .02(1 \mathrm{H}$. d. $J=17.7 \mathrm{~Hz}) .5 .72(1 \mathrm{H} . \mathrm{m}), 6.71(2 \mathrm{H}$. overlap II). $7.11(2 \mathrm{H}$. overlap $\mathrm{m}):{ }^{1.3} \mathrm{C}$ NMR $(75 \mathrm{MHz}$. $\left.\mathrm{CDCl}_{3}\right) \delta 38.7,41.6 .71 .7,116.4,119.0 .124 .2 .127 .5,131.2$. 134.6. 145.1: IR (neat. $\mathrm{cm}^{-1}$ ) 3704. 3418, 3045, 2950; MS(EI) Anal. Calcd for $\mathrm{C}_{11} \mathrm{H}_{1} \mathrm{NO}: 177.11$. Found: 177.00 .

2-[2-(2-Aminophenyl)-2-hydmxyethyl]acrylic acid methy] ester (2c): ${ }^{1} \mathrm{H}$ NMR $\left(300 \mathrm{MHz} . \mathrm{CDCl}_{3}\right) \delta 2.67$ (1H. dd. $J=$ $9.09 \mathrm{~Hz} .13 .9 \mathrm{~Hz}), 2.81(1 \mathrm{H}, \mathrm{dd}, J=3.6 \mathrm{~Hz} .13 .9 \mathrm{~Hz}) .3 .74$ (2H. brs) 3.78 (3H. s). 4.85 (1H. dd. $J=3.63 \mathrm{~Hz} .9 .12 \mathrm{~Hz}$ ). $5.69(1 \mathrm{H}$, d. $J=1 \mathrm{~Hz}), 6.25(1 \mathrm{H} . \mathrm{d} . J=1 \mathrm{~Hz}), 6.65(1 \mathrm{H} . \mathrm{d}, J$ $=7.92 \mathrm{~Hz}), 6.73(1 \mathrm{H}, \mathrm{t}, J=7.47 \mathrm{~Hz}) .7 .08(1 \mathrm{H} . \mathrm{t} . J=7.74$ $\mathrm{Hz}) .7 .16(1 \mathrm{H} . \mathrm{d} . J=6.09 \mathrm{~Hz}):{ }^{13} \mathrm{C}$ NMR $\left(75 \mathrm{MHz} . \mathrm{CDCl}_{3}\right)$ $\delta 39.3 .51 .9,71.0 .116 .2,117.9,126.2,127.4,128.1,128.3$. 136.8. 143.9. 168.0: IR (KBr. cm$\left.)^{-1}\right) 3405.3335 .3246 .2957$.
$1715(-\mathrm{C}=\mathrm{O})$ : MS (El) Anal. Calcd for $\mathrm{C}_{12} \mathrm{H}_{15} \mathrm{NO}_{3}: 221,10$. Found: 221.00

1-(2-Aminophenỵl)-3-methỵlbut-3-en-1-ol (2d): 'H NMR (300 MH $\left.\%, \mathrm{CDCl}_{3}\right) \delta 1.82(3 \mathrm{H}, \mathrm{s}) .2 .47(1 \mathrm{H} . \mathrm{dd} . J=4.05 \mathrm{H} \%$ l0.5 H $\%$ ). $2.73(1 \mathrm{H}$, dd $J=4.5 \mathrm{H} / .9 .87 \mathrm{H} /$ ). $3.60(2 \mathrm{H}$. brs $)$. $4.82(1 \mathrm{H} . \mathrm{dd} J=4.14 \mathrm{H} / . \mathrm{ll} .0 \mathrm{H} /) .4 .89(\mathrm{lH}, \mathrm{s}) .4 .95(\mathrm{lH}$. s) $6.66(1 \mathrm{H}, \mathrm{d} . J=7.86 \mathrm{H} /), 6.73(\mathrm{lH}, \mathrm{t}, J=6.33 \mathrm{H} /) .7 .(09$ $\left(2 \mathrm{H}\right.$, overlap m): ${ }^{13} \mathrm{C}$ NMR $\left(75 \mathrm{MH} \%, \mathrm{CDCl}_{3}\right) \delta 22.8$. 44.4. 71.7. 116.9, 118.2, 127.3,128.1, 128.5, 129.3, 143,0, 145.6: IR (KBr. cm-1) 3365, 3286, 2937, 2997: MS(El) Anal. Calcd for $\mathrm{C}_{11} \mathrm{H}_{15} \mathrm{NO}: 177.11$. Found: 177.10.

1-(2-Amino-6-chlorophenyl)but-3-en-1-ol (2e): ${ }^{3} \mathrm{H}$ NMR (300 $\left.\mathrm{MH} \%, \mathrm{CDCl}_{3}\right) \delta{ }^{\mathrm{l}} \mathrm{H} \mathrm{NMR}\left(300 \mathrm{MH} \% \mathrm{CDCl}_{3}\right) \delta 2.52$ $(\mathrm{lH}, \mathrm{m}), 2.73(\mathrm{lH}, \mathrm{m}), 4.72(2 \mathrm{H}, \mathrm{brs}) .5, \mathrm{l}+(\mathrm{lH}, \mathrm{d} . J=\mathrm{l} 0.3$ $\mathrm{H} / .5 .19(\mathrm{lH}, \mathrm{d} . J=\mathrm{l} 4.0 \mathrm{H} /) .588(\mathrm{lH}, \mathrm{m}) .6 .5(\mathrm{lH}, \mathrm{d} . J=$ $8.04 \mathrm{H} \%) .6 .70(\mathrm{lH} . \mathrm{d} . J=7.89 \mathrm{H} /) .6 .94(1 \mathrm{H} . \mathrm{l} . J=7.98$ $\mathrm{H} \%):{ }^{13} \mathrm{C}$ NMR $\left(75 \mathrm{MH} \% . \mathrm{CDCl}_{3}\right) \delta 38.9 .71 .8 .116 .8 .118 .8$. 124.2. 128.8. 129.0. 134.9. 143.0. 148.0: [R (ncat. $\mathrm{cm}^{-1}$ ) 3475, 3375, 3036. 2947: MS (EI) Anal. Calcd. for $\mathrm{C}_{10} \mathrm{H}_{12} \mathrm{NO}: 197.06$. Found: 196.95.

1-(2-Amino-6-chlorophenỵl)-2-methỵlbut-3-en-1-ol (2f): ${ }^{1} \mathrm{H}$ NMR $\left(300 \mathrm{MH} / . \mathrm{CDCl}_{3}\right) \delta 1.20(3 \mathrm{H} . \mathrm{d} . J=6.78 \mathrm{H} /)$. $3.02(\mathrm{lH} . \mathrm{m}) .3 .38(2 \mathrm{H}$. brs $) .4 .90(\mathrm{lH} . \mathrm{d} . J=16.7 \mathrm{H} /) .4 .95$ $(\mathrm{lH} . \mathrm{d} . J=14.0 \mathrm{H} /), 5.87(\mathrm{lH}, \mathrm{m}), 6.53(\mathrm{lH} . \mathrm{l} . J=7.4 \mathrm{l} \mathrm{H} z)$. $6.72(\mathrm{lH}, \mathrm{d} . J=7.98 \mathrm{H} /) .6 .96(\mathrm{IH}, \mathrm{d} . J=8.73 \mathrm{H} /) \cdot{ }^{13} \mathrm{C}$ NMR (75 $\left.\mathrm{MH} \% . \mathrm{CDCl}_{3}\right) \delta 16.6 .+1.4 .75 .3 .114 .6 .116 .5$. 1 19,0. 120.2. 128,6. 134.0. 140.1. 147.2: IR (KBr. $\left.\mathrm{cm}^{-1}\right)$ 3245.3335, 3066. 2976. 2877: MS (EI) Anal. Calcd. for $\mathrm{C}_{11} \mathrm{H}_{14} \mathrm{CINO}: 211,07$. Found: 211.00 .

2-[2-(2-Amino-6-chlorophenyl)-2-hydroxyethyl]acrylic acid methyl ester (2g): ${ }^{1} \mathrm{H}$ NMR (300 MH $\left.\% . \mathrm{CDCl}_{3}\right) \delta 2.75$ $(\mathrm{lH} . \mathrm{dd} . j=5.43 \mathrm{H} \%$ 13.8 H $\% .3 .02(\mathrm{lH} . \mathrm{dd} . J=8.52 \mathrm{H} \%$ I3.7 H $/$ ). $3.7 \mathrm{l}$ (3H. s). 4.22 (2H. brs). 5.55 (lH. overlap). $5.56(\mathrm{lH} . \mathrm{d} . J=1.4 \mathrm{H} \%) .6 .19(\mathrm{lH} . \mathrm{d} . J=1.35 \mathrm{H} \%) .6 .49(\mathrm{lH}$. d. $J=8.0 \mathrm{l} \mathrm{H} /$ ). $6.66(\mathrm{lH} . \mathrm{d} . J=7.98 \mathrm{H} \%) .6 .92(\mathrm{lH} . \mathrm{t} . J=$ $7.95 \mathrm{H} \%):{ }^{13} \mathrm{C}$ NMR $\left(75 \mathrm{MH} \% . \mathrm{CDCl}_{3}\right) \delta 37.5 .53 .3 .71 .5$. 1]6,1. 1]6.7. 119.2. 124.0. 128.9. 134.1. 137.7. 148.2. 169.0: IR (KBr. cm ${ }^{-1}$ ) 3405. 3296. 3146. 2957. 2847.1561: MS (EI) Anal. Calcd. for $\mathrm{C}_{11} \mathrm{H}_{4+} \mathrm{CINO}$ : 255.06. Found: 255.00 .

1-(2-Amino-6-chlorophenyl)-3-methylbut-3-en-1-ol (2h): ${ }^{1} \mathrm{H}$ NMR (300 MHz. CD $\left.30 D\right) \delta{ }^{1} \mathrm{H}$ NMR (300 MHz. $\left.\mathrm{CD}_{3} \mathrm{OD}\right) \delta 1.79(3 \mathrm{H} . \mathrm{s}) .2 .42(1 \mathrm{H}, \mathrm{ml}) .2 .65(1 \mathrm{H}, \mathrm{ml}) .4 .71$ (1H. s). $4.77(1 \mathrm{H}, \mathrm{s}) .5 .52(1 \mathrm{H}, \mathrm{nl}), 6.61(1 \mathrm{H} . \mathrm{t} . J=8.01 \mathrm{~Hz})$. 6.79 (1H. d. $J=7.8 \mathrm{~Hz}) .7 .10(1 \mathrm{H} . \mathrm{d} . J=7.98 \mathrm{~Hz}):{ }^{13} \mathrm{C}$ NMR $\left(75 \mathrm{MHz} . \mathrm{CD}_{3} \mathrm{OD}\right) \delta 22.9 .42 .7 .70 .1 .116 .0 .124 .8 .129 .0$. 133.0. 142.9. 143.2. 148.2. 151.4: IR $\left(\mathrm{KBr} . \mathrm{cm}^{-1}\right) 3395$. 3325. 3266, 2777: MS (EI) Allal. Calcd. for $\mathrm{C}_{\lrcorner} \mathrm{H}_{\lrcorner} \mathrm{CINO}$ : 211.07 . Found: 211.00 .

1-(2-Amino-5-chlorophenyl)but-3-en-1-ol (2i): 'H NMR (300 MHz, CDCl 3 ) $\delta 2.59(2 \mathrm{H}, \mathrm{ml}) .3 .65$ (2H. brs). $4.63(1 \mathrm{H}$. dd. $J=5.28 \mathrm{~Hz} .8 .31 \mathrm{~Hz}$ ). $5.16(1 \mathrm{H} . \mathrm{d} . J=10.1 \mathrm{~Hz}) .5 .19$ $(1 \mathrm{H} . \mathrm{d} . J=17.0 \mathrm{~Hz}) .5 .82(1 \mathrm{H}, \mathrm{m}), 6.57(1 \mathrm{H}, \mathrm{d} . J=3.21 \mathrm{~Hz})$. 7.02 (2H. overlap): ${ }^{13} \mathrm{C}$ NMR (75 MHz. CDCl 3 ) $\delta 39.6 .72 .4$. 117.8, 118.6. 122.6, 127.1. 128.1. 134.3, 143.4: IR (KBr. $\mathrm{cm}^{-1}$ ) $3345,3226.2917$; $\mathrm{MS}(\mathrm{EI})$ Anal. Calcd for $\mathrm{C}_{10} \mathrm{H}_{1} \mathrm{CINO}$ : 197.06. Found: 197.05 
1-(2-Amino-5-chlorophenyl)-2-methylbut-3-en-1-ol (2j): ${ }^{l} \mathrm{H} \mathrm{NMR}\left(300 \mathrm{MH} / . \mathrm{CDCl}_{3}\right) \delta 1.19(3 \mathrm{H}, \mathrm{d} J=6.6 \mathrm{H} /), 3.64$ $(2 \mathrm{H}$. br s). $4.4 \mathrm{l}(1 \mathrm{H} . \mathrm{d} . J=6.4 \mathrm{H} /) .4 .99(1 \mathrm{H}, \mathrm{d} . J=12 \mathrm{H} z)$. $5.03(\mathrm{lH}, \mathrm{d} . J=17 \mathrm{H} z), 5.72(\mathrm{lH}, \mathrm{m}) .6 .57(\mathrm{lH}, \mathrm{d}, J=3.2 \mathrm{l}$ $\mathrm{H}$ ). $7.02\left(2 \mathrm{H}\right.$. overlap): ${ }^{13} \mathrm{C}$ NMR (75 $\left.\mathrm{MH}_{\iota}, \mathrm{CDCl}_{3}\right) \delta \mathrm{l} 4.9$. 41.6. 76.9. 115.2, 122.5, 126.5, 128.1, 128.9, 140.1, 142.8: IR (KBr. cm- $\mathrm{cm}^{-1}$ 34l5, 3276, 2947, 2847: MS (El) Anal. Calcd for $\mathrm{C}_{11} \mathrm{H}_{1}+\mathrm{CINO}: 2 \mathrm{l} 1.07$. Found: $21 \mathrm{l}$.00.

2-[2-(2-Amino-5-chlorophenỵ)-2-Inyddroxyethỵl]acrỵlic

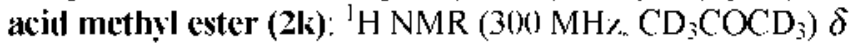
$2.53(1 \mathrm{H}$. dd. $J=9.06 \mathrm{H} / .13 .9 \mathrm{H} z) .2 .74(\mathrm{lH} . \mathrm{dd} J=3.57$ $\mathrm{H} / . \mathrm{l} 4 \mathrm{H} /$ ). $3.09(2 \mathrm{H}$. brs). $3.7 \mathrm{l}(3 \mathrm{H} . \mathrm{s}) .4 .73(\mathrm{lH} . \mathrm{dd} . J=$ $3.42 \mathrm{H} / .9 \mathrm{H} /) ..5 .67(\mathrm{lH} . \mathrm{s}) .6 .2 \mathrm{l}(\mathrm{lH} . \mathrm{s}) .6 .55(\mathrm{lH} . \mathrm{d} . J=$ $8.43 \mathrm{H} /)_{0} 6.96(1 \mathrm{H}, \mathrm{d} J=8.43 \mathrm{H} /) .7 .12(1 \mathrm{H} . \mathrm{s}):{ }^{13} \mathrm{C}$ NMR $\left(75 \mathrm{MH} / . \mathrm{CD}_{3} \mathrm{COCD}_{3}\right) \delta 37.1 .51 .1 .68 .4 .116 .6,121.1 .124$. 126,3, 127.0. 137.1, 140.9. 167.2: IR (KBr. cm-1) 3365. 32 l6. $2986,2827,1696: \mathrm{MS}(\mathrm{El})$ Anal. Calcd. for $\mathrm{C}_{12} \mathrm{H}_{14} \mathrm{ClNO}_{3}$ : 255.06. Found: 255.01 .

1-(2-Amino-5-chlorophenyl)-2-methylbut-3-en-1-ol (2l): ${ }^{l} \mathrm{H}$ NMR (300 MH $\left.\% . \mathrm{CDCl}_{3}\right) \delta$ l.81 (3H. s). 2.43 (lH. dd. $J$ $=3.57 \mathrm{H} \% 13.8 \mathrm{H} \%) \cdot 2.66(\mathrm{lH} . \mathrm{dd} . J=10.1 \mathrm{H} \%, 13.9 \mathrm{H} \%) .3 .6$ (2H. brs) 4.75 (l H. dd. $J=3.84 \mathrm{H} \% .9 .96 \mathrm{H} \%$ ). 4.88 (l H. s). $4.96(1 \mathrm{H} . \mathrm{s}) .6 .57(\mathrm{lH} . \mathrm{d} . J=8.7 \mathrm{H} /) .7 .18(\mathrm{lH} . \mathrm{d} . J=2.4$

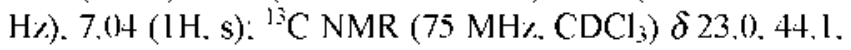
71.5. 118,1, 119,0, 127.3. 129,1, 129,3, 142,8. 144.3: IR (KBr. $\mathrm{cm}^{-1}$ ) 3455. 3355. 3036. 2927: MS (EI) Anal, Calcd. for $\mathrm{C}_{11} \mathrm{H}_{1+} \mathrm{CINO}: 211.07$. Found: 211,00 .

1-(6-Aminobenzo[1,3] dioxol-5-yl)but-3-en-1-ol (2m): ${ }^{1} \mathrm{H}$ NMR (300 MH\%, CDCl $) \delta 2,6 \mathrm{l}(\mathrm{lH}, \mathrm{m}) .4,67(\mathrm{lH}, \mathrm{dd} . J=$ $5.49 \mathrm{H} / .8 .25 \mathrm{H} /$ ). $5.14(\mathrm{lH} . \mathrm{d} . J=7.4 \mathrm{H} /$ ). 5.19 (l H. d. $J=$ $11.0 \mathrm{H} /$ ). 5.79 (l H.m). $5.83(2 \mathrm{H} . \mathrm{s}) .6 .28(\mathrm{lH} . \mathrm{s}) .6 .62(\mathrm{lH}$. s): ${ }^{1} \mathrm{C}$ NMR $\left(75 \mathrm{MH} \% \mathrm{CDCl}_{3}\right) \delta+0.1,72.0 .98 .8,100,6$. 107.2. 118.3, 134.7, 139.7, 144.3, 147,8, 149,2: IR (ncal. $\mathrm{cm}^{-1}$ ) 3330. 3250. 2978: MS (El) Anal, Calcd. for $\mathrm{C}_{11} \mathrm{H}_{3} \mathrm{NO}_{3}: 207.08$. Found: 207.06 .

1-(6-Aminobenzo|1,3]dioxol-5-yl)-2-methylbut-3-en-1ol (2n): ${ }^{1} \mathrm{H}$ NMR $\left.(300) \mathrm{MH} \%, \mathrm{CDCl}\right) \delta \mathrm{l}, \mathrm{l}+(3 \mathrm{H}, \mathrm{d} . J=6,66$ Hz). $2.81(1 \mathrm{H} . \mathrm{m}) .3 .30(2 \mathrm{H}$, brs). $4.40(1 \mathrm{H}, \mathrm{d} . J=7.44 \mathrm{~Hz})$. $4.97(1 \mathrm{H} . \mathrm{d} . J=9.9 \mathrm{~Hz}) .5 .11(1 \mathrm{H} . \mathrm{d} . J=17.1 \mathrm{~Hz}), 5.80(1 \mathrm{H}$. m) .5 .84 (2H. s) 6.23 (1H. s), 6.58 (1H. s): ${ }^{13} \mathrm{C}$ NMR $(75$ $\left.\mathrm{MHz}_{\mathrm{CDCl}}\right) \delta 17.1,42.7,77.6,100.5 .108 .9 .114 .8,119.6$. 139.7. 140.1, 140.5, 146.9. IR (neat, $\mathrm{cm}^{-1}$ ) 3350, 3255. 2976: MS (EI) Anal. Calcd. for $\mathrm{C}_{12} \mathrm{H}_{15} \mathrm{NO}_{3}: 221.10$. Found: 221.00 .

2-[2-(6-Aminobenzo[1,3]dioxol-5-yl)-2-hydroxyethyl]acrylic acid methyl ester (20): ${ }^{1} \mathrm{H}$ NMR (300 MHz. $\left.\mathrm{CDCl}_{3}\right)$ $\delta 2.62(1 \mathrm{H}$. dd $J=9.06 \mathrm{~Hz} .13 .9 \mathrm{~Hz}) .2 .78(1 \mathrm{H}$. dd $J=3.54$ Hz, 17.4 Hz) .3 .70 (3H. brs). 4.80 (1H. dd. $J=3.57 \mathrm{~Hz}, 9.06$ $\mathrm{Hz}) .5 .71(1 \mathrm{H}, \mathrm{s}) .5 .82(2 \mathrm{H}, \mathrm{s}) .6 .24(1 \mathrm{H} . \mathrm{s}) .6 .25(1 \mathrm{H}, \mathrm{s})$. $6.72(1 \mathrm{H}, \mathrm{s}){ }^{1.3} \mathrm{C}$ NMR $\left(75 \mathrm{MHz} . \mathrm{CDCl}_{3}\right) \delta 39.9 .52 .0 .70 .3$. 98.4, 100.5. 106.3, 120.0. 128.3, 136.8. 138.6, 140.0. 147.1. 168.0: IR (neat. $\mathrm{cm}^{-1}$ ) $3385,3256,2976.1688,1646$ : MS (EI) Anal Calcd for $\mathrm{C}_{13} \mathrm{H}_{3} \mathrm{NO}_{3}: 265.09$. Found: 265.00 .

1-(6-Aminobenzo[1,3|dioxol-5-y])-3-methylbut-3-en-1ol (2p): ' $\mathrm{H}$ NMR (300 MHz, CDCl ) $_{3} \delta 1.75$ (3H. s). 2.43 (1H. dd $J=4.14 \mathrm{~Hz} . J=13.6 \mathrm{~Hz}), 2.66(1 \mathrm{H} . \mathrm{dd}, J=9.96$ Hz $J=10.7 \mathrm{~Hz}$ ) 3.5 (2H. brs) 4.75 (1H. dd. $J=3.96 \mathrm{~Hz}$.
$9.66 \mathrm{H} /) .4 .87(1 \mathrm{H}, \mathrm{s}) .4 .93(1 \mathrm{H}, \mathrm{s}) .5 .81(2 \mathrm{H}, \mathrm{s}) .6 .25(1 \mathrm{H}$, s). $6.62(1 \mathrm{H}, \mathrm{s}):{ }^{3} \mathrm{C}$ NMR $\left(75 \mathrm{MH} \% \mathrm{CDCl}_{3}\right) \delta 22.3 .44 .0$. 70.1,98.8, 100.6. 107.1, 113.9. 119.2. 139.9. 142.4. 146.9. 147.1: IR (ncat. $\mathrm{cm}^{-1}$ ) 3455, 3345, 2996, 2847: MS (EI) Anal. Calcd for $\mathrm{C}_{12} \mathrm{H}_{15} \mathrm{NO}_{3}: 221$.10. Found: 221.00 .

1-(3-Methoxy-2-nitrophenyl)but-3-en-1-ol (3a): ${ }^{1} \mathrm{H}$ NMR (300 MH $\left./, \mathrm{CDCl}_{3}\right) \delta 2.56(2 \mathrm{H}, \mathrm{m}), 3.67(3 \mathrm{H}, \mathrm{s}), 4.72(\mathrm{lH}$. dd $J=5.37 \mathrm{H} / .8 .46 \mathrm{H} /) .5 .12(\mathrm{lH} . \mathrm{d} J=9.2 \mathrm{H} /) .5 .16(\mathrm{lH}$. d. $J=17.0 \mathrm{H} \%), 5.79(1 \mathrm{H}, \mathrm{m}), 6.70-6.74(3 \mathrm{H}, \mathrm{m}):{ }^{13} \mathrm{C}$ NMR $\left(75 \mathrm{MH} \%, \mathrm{CDCl}_{3}\right) \delta 39.7,55.5,72,6,109.3,117,2,118.5$. 1 19.3, 131,0, 133.5, 134.9, 147.5: IR (ncat. $\mathrm{cm}^{-1}$ ) 3365 $(-\mathrm{OH}) .2907$ (aromatic $\mathrm{C}-\mathrm{H}), 1541,1399(-\mathrm{N}=\mathrm{O})$.

1-(3-Methoxy-2-nitrophenyl)-2-methỵlbut-3-en-1-ol (3b): ${ }^{1} \mathrm{H}$ NMR $\left.\left(300 \mathrm{MH} /, \mathrm{CDCl}_{3}\right) \delta 1.15(3 \mathrm{H}, \mathrm{d} . J=6.7 \mathrm{H} /) .2 .79\right)$ $(\mathrm{lH}, \mathrm{m}) .3 .81(3 \mathrm{H}, \mathrm{s}) .4 .49(\mathrm{lH} . \mathrm{d} . J=7.2 \mathrm{H} /$ ). $4.95(\mathrm{lH} . \mathrm{d} . J$ $=8.9 \mathrm{H} z), 5.03(\mathrm{lH} \cdot \mathrm{d} . J=17,1 \mathrm{H} /), 5.71(1 \mathrm{H}, \mathrm{m}), 6.65-6.72$ (3H. m): ${ }^{13} \mathrm{C}$ NMR (75 $\left.\mathrm{MH} \%, \mathrm{CDCl}_{3}\right) \delta 15.0,41.7 .55 .4$. 78.2. 108.9, 1]4.6. 116.6. 120.4, 134.0. 140.5. 147.5. 154.2: IR (ncal. $\mathrm{cm}^{-1}$ ) $3435,3395,3076.2937,1501,1277$.

2-[2-Hydroxy-2-(3-methoxy-2-nitrophenyl)ethyl]acrylic acid methỵl ester (3c): ' $\mathrm{H}$ NMR $\left(300 \mathrm{MH} \% \mathrm{CDCl}_{3}\right) \delta 2.67$ $(\mathrm{lH} . \mathrm{dd} . J=9 .(\mathrm{N}) \mathrm{H} / . . J=14.0 \mathrm{H} \%$ ). $2.8 \mathrm{l}(\mathrm{lH} . \mathrm{dd} . J=3.63$ $\mathrm{H} / . J=14.0 \mathrm{H} /$ ). $3.7(3 \mathrm{H} . \mathrm{s}) .3 .83(3 \mathrm{H} . \mathrm{s}) .4 .86(\mathrm{lH} . \mathrm{dd} . J=$ $3.57 \mathrm{H} \% . J=9.06 \mathrm{H} /$ ). $5.68(\mathrm{lH} . \mathrm{s}) .6 .23(\mathrm{lH} . \mathrm{s}) .6 .70-6.80$ $\left(2 \mathrm{H}\right.$. overlap H). $6.8 \mathrm{I}(\mathrm{lH} . \mathrm{d} J=7.2 \mathrm{H} /)^{13} \mathrm{C}$ NMR $(75$ $\left.\mathrm{MH} \% \mathrm{CDCl}_{3}\right) \delta 22.2 .43 .5,55.5 .70 .9$. 109.3. 113.6. I17.1. 1 19.2. 126.9. 134.6. 142.5. 147.5: IR (KBr. $\left.\mathrm{cm}^{-1}\right) 3490$. $3390.2744,1496,1297.1222$.

1-(3-Methoxy-2-nitrophenyl)-3-methỵlbut-3-en-1-ol (3d): ${ }^{\mathrm{l}} \mathrm{H}$ NMR $\left(300 \mathrm{MH} / . \mathrm{CDCl}_{3}\right) \delta \mathrm{I} .8 \mathrm{I}(3 \mathrm{H} . \mathrm{s}) .2 .46$ (l H. dd. $J$ $=3.84 \mathrm{H} \% . J=14.0 \mathrm{H} /) ..2 .73(\mathrm{lH} . \mathrm{dd} . J=10.0 \mathrm{H} / .13 .9 \mathrm{H} /)$. $3.84(3 \mathrm{H}, \mathrm{s}) .3 .84(\mathrm{lH} . \mathrm{dd} . J=4.11 \mathrm{H} / .9 .87 \mathrm{H} /) .488(\mathrm{lH}$. s). 4.93 (1 H. s). 6.68-6.76 (3H. ovcrlap): ${ }^{13} \mathrm{C}$ NMR (75 MH\%. $\left.\mathrm{CDCl}_{3}\right) \delta 22.2 .43 .5 .55 .5 .70 .9 .109 .3 .113 .6 .117 .1 .119 .2$. 126.9. 134.6. 142.5. 147.5. 154.2: IR (ncat. $\mathrm{cm}^{-1}$ ) 3176. $2907.1501(-\mathrm{N}=\mathrm{O}) .1247(-\mathrm{N}=\mathrm{O})$.

Representative intramolecular cyclization procedure: Synthesis of 6a.

2-\{2-Hydroxy-2-[2-(p-toluenesulfonylamino phenyl]ethyl\}acrylic acid methyl ester (4a): To a stirred solution of $\mathbf{2 c}$ (51.4 mg, $0.23 \mathrm{mmol}$ ) in $3 \mathrm{~mL}$ of pyridine was added TsCl (88.6 $\mathrm{mg} .0 .46 \mathrm{mmol}$ ) under $\mathrm{N}_{2}$ atmosphere. The reaction mixture was stirred at room temperature for about $12 \mathrm{~h}$. The mixture was poured into the cooled water. and extracted with methylene chloride. The combined organic layer was dried $\left(\mathrm{MgSO}_{4}\right)$. concentrated and purified over silica gel to give $87.4 \mathrm{mg}(78 \%)$ of tosylate. ${ }^{1} \mathrm{H}$ NMR (CDCl. $\left.300 \mathrm{MHz}\right) \delta$ $2.33(3 \mathrm{H}, \mathrm{s}), 2.47(2 \mathrm{H} . \mathrm{d}, J=6.48 \mathrm{~Hz}) .3 .35(1 \mathrm{H}, \mathrm{d} . J=3.18$ Hz) $3.76(3 \mathrm{H} . \mathrm{s}) .4 .78(1 \mathrm{H} . \mathrm{nl}) .5 .44$ (1H.s). 6.15 (1H. d. $J=$ $1.2 \mathrm{~Hz}), 6.98-7.17(3 \mathrm{H}$, overlap H). $7.18(2 \mathrm{H}, \mathrm{d} . J=8.01$ Hz). $7.42(1 \mathrm{H}, \mathrm{d}, J=7.95 \mathrm{~Hz}) .7 .67(2 \mathrm{H}, \mathrm{d} . J=8.25 \mathrm{~Hz})$. $8.58(1 \mathrm{H}, \mathrm{s}) ;{ }^{1.3} \mathrm{C} \mathrm{NMR}\left(\mathrm{CDCl}_{3 .}, 75 \mathrm{MHz}\right) \delta 21.9 .41 .2 .52 .7$. $60.8,122.2 .124 .9,127.5 .127 .6,128.7 .129 .4,130.0,133.3$. 135.5. 136.6. 137.4. 144.1: IR (neat. $\mathrm{cm}^{-1}$ ) 3482. 3238 . $1718.1710,1340,1158,928$.

2- \{2-0xo-2-[2-(p-toluenesulfonylamino)phenyl]ethy]\} acrylicacid methyl ester (5a): To a stirred solution of 4 a 
(33.6 mg. $0.0895 \mathrm{mmol}$ ) in $10 \mathrm{~mL}$ of $\mathrm{CH}_{2} \mathrm{Cl}_{2}$. was added 20 $\mathrm{mg}$ of silica gel and PCC (38.6 mg. $0.179 \mathrm{mmol})$. Aficr stirring for $16 \mathrm{~h}$ at room temperature. the reaction mixture was filtered through celite pad. The solvent was removed in vocto. The residuc was purificd by flash chromatography over silica gel 10 yield $28 \mathrm{mg}$ ( $84 \%$ ) of product. 'H NMR $\left(\mathrm{CDCl}_{3} 300 \mathrm{MH} z\right) \delta 2.19\left(3 \mathrm{H}_{\mathrm{s}} \mathrm{s}\right) .3 .87\left(3 \mathrm{H}_{\mathrm{s}} \mathrm{s}\right) .3 .98(2 \mathrm{H} . \mathrm{s})$. 5.63 (1H. s). 6.4 (1H. s). 7.0-7.88 (8H. overlap H). 11.25 (1H. s): ${ }^{13} \mathrm{C}$ NMR $\left(\mathrm{CDCl}_{3 .}, 75 \mathrm{MH} /\right.$ ) $\delta$ 14.6, 43, 1, 52.6. 119.3. 123.0. 124.9. 127.6, 129.3, 130.0, 131.6, 133.2, 134.4. 136.6. 140.6. 140.3. 167.1: IR (ncat. $\mathrm{cm}^{-1}$ ) 3124. 1726. 1650. 1334. 1160 .

2-[3-Oxo-1-( $p$-toluenesulfonyl)-2,3-dihỵdro- $1 H$-indol2-yl]propionic acid methyl ester (6a): To a stirred solution of 5 a $(63.6 \mathrm{mg} .0 .14 \mathrm{mmol})$ in $3 \mathrm{~mL}$ of methy lene chloride was added $60 \mathrm{~mL}(0.34 \mathrm{mmol})$ of DIPEA. After stirring for 4 hour at $\mathrm{rl}$. the reaction mixture was quenched by $\mathrm{l} \mathrm{mL}$ of water and extracted with $\mathrm{CH}_{2} \mathrm{Cl}_{2}$. The organic layer was dried concentrated. and purified over silica gel to give 47 mg $(88 \%)$ of dihy droindolone product $6 \mathbf{a}^{1}{ }^{1} \mathrm{H}$ NMR $\left(\mathrm{CDCl}_{3}\right.$. $300 \mathrm{MH} /) \delta 1.26(3 \mathrm{H} . \mathrm{d} . J=9.03 \mathrm{H} \%) .2 .35(3 \mathrm{H} . \mathrm{s}) .3 .67$ $(1 \mathrm{H}, \mathrm{m}) .3 .73(3 \mathrm{H} . \mathrm{s}) .4 .22(\mathrm{lH}, \mathrm{d} . J=2.46 \mathrm{H} /) .7 .20 \mathrm{l}-8 . \mathrm{l}$ (8H. overlap $\mathrm{H}$ of another isomer): ${ }^{13} \mathrm{C}$ NMR $\left(\mathrm{CDCl}_{3} .75\right.$ MH॰) $\delta 11.6 .21 .9 .43 .4 .52 .6 .68 .1 .117 .6 .124 .6 .125 .2$. 125.7. 127.8. 130.4. 130.5. 137,5. 145,6. 153,7. $173,7$. 197.3.: IR (neat. $\mathrm{cm}^{-1}$ ) 1724. 1602.1364. 1174.: HRMS (E]) Anal. Calcd. for $\mathrm{C}_{19} \mathrm{H}_{19} \mathrm{NO}_{5} \mathrm{~S}: 373.0984$. Found: 373.0991 .

2-[4-Chloro-3-oxo-1-( $p$-toluenesulfonyl)-2,3-dihydro$1 \mathrm{H}$-indol-2-yl]propionic acid methyl ester (6b): 'H NMR $\left(\mathrm{CDCl}_{3} .300 \mathrm{MH} \%\right) \delta 1.32(3 \mathrm{H} . \mathrm{d} . J=7.26 \mathrm{H} / 2.2 .37(3 \mathrm{H} . \mathrm{s})$. $3.50(\mathrm{lH} . \mathrm{m}) .3 .72(3 \mathrm{H}, \mathrm{s}) .4 .22(\mathrm{lH} . \mathrm{d} . J=2.49 \mathrm{H} /) .7 .13$ (1H. d. $J=8.19 \mathrm{H} /$ ). $7.23(3 \mathrm{H}$. overlap of prolon). 7.52-7.62 (4H. overlap of protons): ${ }^{13} \mathrm{C}$ NMR $\left(\mathrm{CDCl}_{3} .75 \mathrm{MH} /\right.$ ) $\delta 1 \mathrm{l}, 4$. 21.5. 43.3. 52.2. 67.8. 115.2. 126.1. 127.4. 130.1. 132.1. 132.4. 136.9. 145.5. 154.6. 173.1. 194.0: IR (ncal. $\mathrm{cm}^{-1}$ ) 1730. 1590. 1366. 1174: HRMS (EI) Anal. Caled for $\mathrm{C}_{19} \mathrm{H}_{18} \mathrm{CINO}_{5} \mathrm{~S}: 409,0565$. Found: 409,0560 .

2-[5-Chloro-3-oxo-1-( $p$-toluenesulfony])-2,3-dihydro$1 H$-indol-2-yl]propionic acid methyl ester (6c): 'H NMR $\left(\mathrm{CDCl}_{3}, 300 \mathrm{MHz}\right) \delta 1.30(3 \mathrm{H} . \mathrm{d} . J=6.48 \mathrm{~Hz}) .2 .37(3 \mathrm{H.} \mathrm{s})$. $3.52(1 \mathrm{H} . \mathrm{m}) .3 .71(3 \mathrm{H} . \mathrm{s}) .4 .15(1 \mathrm{H} . \mathrm{d} . J=2.64 \mathrm{~Hz}) .7 .23-$ $8.06\left(7 \mathrm{H}\right.$ overlap with isomer respectively): ${ }^{13} \mathrm{C}$ NMR $\left(\mathrm{CDCl}_{3}, 75 \mathrm{MHz}\right) \delta 12.1,21.9 .43 .8 .52 .7 .68 .5$. 118.9. 124.1 . $127.8 .130 .5,131.2,132.8 .137 .6,145.9 .151 .9 .173 .6,196.1$; IR (neat. $\mathrm{cm}^{-1}$ ) 1728. 1602. 1366. 1174. 1130: HRMS (EI) Anal. Calcd. for $\mathrm{C}_{10} \mathrm{H}_{1} \mathrm{ClNO}_{5} \mathrm{~S}: 407.0594$. Found: 407.0591 .

2-Methyl-1-( $p$-toluenesulfonyl)-2,3-dihydro-1 $H$-quinolin4-one (7d): ${ }^{\mathrm{H}} \mathrm{NMR}\left(\mathrm{CDCl}_{3}, 300 \mathrm{MHz}\right) \delta 1.26(3 \mathrm{H}, \mathrm{d} . J=$ $6.45 \mathrm{~Hz}$ ). 2.23 ( $1 \mathrm{H} . \mathrm{d} . J=19.4 \mathrm{~Hz}$ ). 2.29 (1H. overlap). 2.38 $(3 \mathrm{H} . \mathrm{s}) .4 .89(1 \mathrm{H}, \mathrm{m}) .7 .21(2 \mathrm{H}, \mathrm{d} . J=6.3 \mathrm{~Hz}) .7 .29(2 \mathrm{H}$. overlap m). $7.55(2 \mathrm{H} . \mathrm{d} . J=12.9 \mathrm{~Hz}$ ). 7.60 ( lH. t. $J=8.55$ $\mathrm{Hz}) .7 .91(1 \mathrm{H} . \mathrm{t} . J=8.28 \mathrm{~Hz}):{ }^{13} \mathrm{C} \mathrm{NMR}\left(\mathrm{CDCl}_{3} .75 \mathrm{MHz}\right) \delta$ $19.5,21.5,41.9,51.8,125.3,125.6,126.3 .126 .8,127.0$. 130.0. 134.9,136.5, 139.6. 144.4. 192.4.: IR (neat $\mathrm{cm}^{-1}$ ) 1688. 1350, 1168: HRMS (EI) Anal. Calcd for $\mathrm{C}_{17} \mathrm{H}_{17} \mathrm{NO}_{3} \mathrm{~S}$ : 315.0929. Found: 315.0929

6-Chloro-2-methyl-1-( $p$-toluenesulfonyl)-2,3-dihydro-
$\boldsymbol{H}$-quinolin-4-one (7e): ' $\mathrm{H}$ NMR ( $\left.\mathrm{CDCl}_{3} .300 \mathrm{MH} /\right) \delta$ $1.27(3 \mathrm{H}, \mathrm{d} . J=6.9 \mathrm{H} r) .2 .25(1 \mathrm{H} . \mathrm{d} . J=1.83 \mathrm{H} r) .2 .29(1 \mathrm{H}$. d. $J=5.52 \mathrm{H} /), 2.36(3 \mathrm{H} . \mathrm{s}) .4 .87(1 \mathrm{H}, \mathrm{m}) .7 .23(2 \mathrm{H} . \mathrm{d} . J=$ $8.19 \mathrm{H} /) .7 .5 \mathrm{l}(2 \mathrm{H} . \mathrm{d} . J=6.15 \mathrm{H} / 2.7 .54(\mathrm{lH} . \mathrm{d} . J=9.15$ $\mathrm{H} /) .7 .87(1 \mathrm{H}, \mathrm{d} . j=8.01 \mathrm{H} \%) .7 .88(1 \mathrm{H} . \mathrm{s}):{ }^{13} \mathrm{C}$ NMR $\left(\mathrm{CDCl}_{3}, 75 \mathrm{MH}.\right) \delta 19.4,21,5,41.5,51.8,77,1,126,1.126,6$. $126.8,127.8,130.1,131.6,134,6,136.2,138,0.144,6,191.2$ : IR (neat. $\mathrm{cm}^{-1}$ ) 1694. 1470. 1354. 1 166.: HRMS (EI) Anal. Calcd for $\mathrm{C}_{17} \mathrm{H}_{16} \mathrm{ClNO}_{3} \mathrm{~S}: 349.0539$. Found: 349.(5539.

2,3-Dimethyl- 1-( $p$-toluenesulfonyl)-2,3-dihydro- $1 \mathrm{H}$ quinolin-4-one (7f): 'H NMR ( $\mathrm{CDCl}_{3}, 300 \mathrm{MH} /$ ) $\delta 1.05$ $(3 \mathrm{H} . \mathrm{d} . J=6.84 \mathrm{H} /) .1 .16(3 \mathrm{H} . \mathrm{d} . J=6.9 \mathrm{H} \%) .2 .38(3 \mathrm{H} . \mathrm{s})$. $2.54(\mathrm{lH}, \mathrm{m}) .4 .79(\mathrm{lH}, \mathrm{m}) .7 .19(4 \mathrm{H}$, ovcrlap). $7.57(\mathrm{lH}, \mathrm{t} . J$ $=9.15 \mathrm{H} / \mathrm{s} .7 .59(2 \mathrm{H}, \mathrm{d} . J=8.25 \mathrm{H} / \mathrm{s} .7 .90(\mathrm{lH} . \mathrm{l} . J=8.37$ $\mathrm{H} \%):{ }^{13} \mathrm{C}$ NMR $\left(\mathrm{CDCl}_{3 .} 75 \mathrm{MH} /\right) \delta 11.2 .13 .8 .21 .5 .+4.2$. 57.3, 124.8. 124.9. 124.99, 126,7, 127.2. 129.9, 134,6. 137.1. 139.7. 144.3. 195.2: IR $\left(\mathrm{KBr} . \mathrm{cm}^{-1}\right)$ l684. 1596. 1356. $1166:$ HRMS (EI) Anal. Calcd. for $\mathrm{C}_{18} \mathrm{H}_{19} \mathrm{NO}_{3} \mathrm{~S}: 32 \%$. 1086 . Found: 329.1074 .

6-Chloro-2,3-dimethyl-1-( $p$-toluenesulfonyl)-2,3-dihydivo$1 \mathrm{H}$-quinolin-t-one (7g): ${ }^{1} \mathrm{H}$ NMR $\left(\mathrm{CDCl}_{3} .300 \mathrm{MH} /\right) \delta$ $1.04(3 \mathrm{H} . \mathrm{d} . J=6.9 \mathrm{H} /), \mathrm{l}, \mathrm{l} 2(3 \mathrm{H} . \mathrm{d} . J=6.24 \mathrm{H} / ., 2.39(3 \mathrm{H}$. s). $2.48(\mathrm{lH} . \mathrm{m}) .4 .76(\mathrm{lH} . \mathrm{m}) .7 .25(2 \mathrm{H} . \mathrm{d} . J=8.07 \mathrm{H} / 2)$. 7.49 (lH. d. $J=2.52 \mathrm{H} /$ ). 7.59 (2H. d. $J=8.25 \mathrm{H} /$ ). 7.87 (3H. overlap): ${ }^{13} \mathrm{C}$ NMR $\left(\mathrm{CDCl}_{3 .} 75 \mathrm{MH} /\right) \delta 11.5,14.2$. 22.0. 44.6. 57.8. 126.1. 126.9. 127.1. 127.2. 130.5. 131.5. 134.8. 137.2. 138.6. 145.0. 194.6: IR (KBr. $\left.\mathrm{cm}^{-1}\right) 1694$. 1594. 1354. 1 164.: HRMS (EI) Anal. Calcd for $\mathrm{C}_{18} \mathrm{H}_{18} \mathrm{CINO}_{3} \mathrm{~S}$ : 363.0696 . Found: 363.069$)$.

2,2-Dimcthyl-1 - ( $p$-toluenesulfonyl)- 2,3-dihydro- $1 \mathrm{H}$ quinolin-4-one (7h): To a stirred solution of 5h $(20.8 \mathrm{mg}$. $0.06 \mathrm{mmol}$ ) in $3 \mathrm{~mL}$ of methy lene chloride was added $18 \mathrm{~mL}$ (0. $12 \mathrm{~mm}$ l) of DBU. After stirring for $50 \mathrm{~h}$ at reflux. the reaction mixture was quenched by $1 \mathrm{~mL}$ of water and cxtracted with $\mathrm{CH}_{2} \mathrm{Cl}_{2}$. The organic layer was dried. concentratcd. and purificd over silica gel to give $48 \mathrm{mg}$ $(70 \%)$ of the cyclized product 7 h. ${ }^{1} \mathrm{H}$ NMR $\left(\mathrm{CDCl}_{3} .300\right.$ MHz) $\delta 1.46(6 \mathrm{H} . \mathrm{s}) .2 .29(2 \mathrm{H} . \mathrm{s}) .2 .42(3 \mathrm{H} . \mathrm{s}) .7 .26(2 \mathrm{H.} \mathrm{d}$. $J=7.6 \mathrm{H} /) .7 .44(2 \mathrm{H} . \mathrm{d} . J=7.54 \mathrm{H} /) .7 .56(\mathrm{lH} . \mathrm{d} . J=7.7$ H\%). $7.70\left(\mathrm{lH}\right.$. d. $J=8.6 \mathrm{H} \%$ ). $7.94(\mathrm{lH} . \mathrm{s})$ : $^{1.7} \mathrm{C} \mathrm{NMR}(\mathrm{CDCl}$. $75 \mathrm{MHz}) \delta 21.4 .21 .5 .27 .9 .48 .9 .60 .4,121.7 .122 .7,126.9$. 129.5. 130.7, 134.1, 142.7. 144.1. 194.1: IR (neat. $\mathrm{cm}^{-1}$ ) 1690. 1598. 1354. 1162.: HRMS (EI) Anlal. Calcd for $\mathrm{C}_{18} \mathrm{H}_{10 \mathrm{NO}} \mathrm{S}: 329.1086$. Found: 329.1091 .

6-Chloro-2,2-dimethyl-1-(p-toluenesulfonyl)-2,3-dihydro1H-quinolin-4-one (7i): ' $\mathrm{H}$ NMR (CDCl $300 \mathrm{MHz}) \delta 1.44$ (6H.s). $2.25(2 \mathrm{H} . \mathrm{s}) .2 .41(3 \mathrm{H} . \mathrm{s}) .7 .24(2 \mathrm{H} . \mathrm{d} . J=7.56 \mathrm{~Hz})$. $7.43(2 \mathrm{H}$. d. $J=7.68 \mathrm{~Hz}) .7 .57(1 \mathrm{H} . \mathrm{d} . J=7.71 \mathrm{~Hz}) .7 .70$ (1H. d. $J=8.79 \mathrm{~Hz}), 7.90(1 \mathrm{H} . \mathrm{s}):{ }^{13} \mathrm{C}$ NMR $\left(\mathrm{CDCl}_{3} .75\right.$ MHz) $\delta 22.0,29.7 .30 .0 .49 .0 .609 .126 .6 .127 .6,129.9$. 130.0. 130.5, 132.3, 133.5, 134.4. 138.4. 141.6, 144.8. 193.3. IR (neat. $\mathrm{cm}^{-1}$ ) 1692. 1466. 1356. 1164: HRMS (EI) Anal. Calcd. for $\mathrm{C}_{18} \mathrm{H}_{1} \mathrm{CINO}_{3} \mathrm{~S}: 363.0696$. Found: 363.0691 .

Acknowledgment. This work was supported by grants from "Critical Teclunology 21 " of the Korea Ministry of Science and Teclunolog: 


\section{References}

1. (a) Reddy: G. V: Rao. G. V: Iyengar. D. S. Tethahedron tett. 1999, t0. 3937. (b) for retiens. see: i) Larock. R. C. Comprehensive Onganic Ifomsformatons: VCH I: Nen York. 1989:

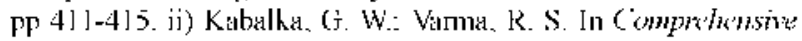
Orgunic Swhinsis: Trost. B. M.. Fleming. I.. Eds.: Pergamon Press: Oxford. 1991: Vol. 8. pp 363-379. iii) Sauvé. G.: Rao. V. S. In Comprethensive Onganic finctional (Homp Thonsfomations. Katrickky, A. R: Melh-Cohn, O: Rees, C. W. Iids: Pergamon Press: (Nord 1995: Vol. 2. pp 737-817.

2. Jor reviews, see: (a) I.j, C. 1.: Chan. T. I1. Orgmic Reaction in Aqraens Media: John Wiley \& Sons: New York. 1997. (b) Li. C. I. Tuthledron 1996. 52. 5643. (c) Chan. T. H.: Isaac. M. B. Pur' 1ppl Chem. 1996. 68. 919. (d) Lubineau. A.: Auge. J.: Quencau. Y. Synthesis 1994. 741. (e) Lubineau. A.: Auge J : Queneatu, Y. In

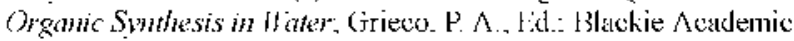
\& Prolessional: 1.ondon. 1998. (f) Paquelte, I. A. In (hesen Chenistry-Fontior in Bonign Chatical Symheses and Precesses: Anastas. P. T. Williamson. T. C.. Eds.: Ostord University Press: New York. 1998. (g) Li. C. J. Chem. Rev 1993. 93. 2623, (h) Li. C. J.: Chan. I. H. Tetrahedron 1999. 55. 11149.

3. Moods C. J. Pitts, M. P. Swhtett 1998, 1028

4. I ee J (G.: Choi, K. I.: Koh. H. Y. Kim, Y. S.: Kang. Y. II.; Cho. Y. S. Symlksis 2001. I.81.

5. Cha. J. H.: Pac. A. N.: Choi. K. I.: Cho. Y. S.: Kolı. H. Y.: Lee. E. J. Chem. Soc. Pey th Trons 1 2001. 2079. 